\title{
Real time monitoring of the Bragg-peak position in ion therapy by means of single photon detection
}

M. Testa ( $₫)$, M. Bajard, M. Chevallier, D. Dauvergne, P. Henriquet, F. Le Foulher, C. Ray, E. Testa

IPNL, Université de Lyon, F-69003 Lyon; Université Lyon 1 and CNRS/IN2P3, UMR 5822, F69622 Villeurbanne, France. e-mail: m.testa@ipnl.in2p3.fr. Phone +3304 72448463 . Fax +33 0472431452

N. Freud, J.M. Létang, M-H. Richard*

Laboratoire de Contrôles Non Destructifs par Rayonnements Ionisants, INSA-Lyon, F-69621 Villeurbanne cedex, France

S. Karkar

CPPM, Aix-Marseille Université, CNRS/IN2P3, Marseille, France

R. Plescak, D. Schardt

GSI, D-64291, Darmstadt, Germany

* M-H. Richard is also with IPNL, Université de Lyon, F-69003 Lyon; Université Lyon 1 and CNRS/IN2P3, UMR 5822, F-69622 Villeurbanne, France 


\begin{abstract}
For real-time monitoring of the longitudinal position of the Bragg-peak during an ion therapy treatment, a novel non-invasive technique has been recently proposed that exploits the detection of prompt $\gamma$-rays issued from nuclear fragmentation. Two series of experiments have been performed at the GANIL and GSI facilities with $95 \mathrm{MeV} / \mathrm{u}$ and $305 \mathrm{MeV} / \mathrm{u}^{12} \mathrm{C}^{6+}$ ion beams stopped in PMMA and water phantoms. In both experiments a clear correlation was obtained between the carbon ion range and the prompt photon profile. Additionally, an extensive study has been performed to investigate whether a prompt neutron component may be correlated with the carbon ion range. No such correlation was found. The present paper demonstrates that a collimated set-up can be used to detect single photons by means of time-offlight measurements, at those high energies typical for ion therapy. Moreover, the applicability of the technique both at cyclotron and synchrotron facilities is shown. It is concluded that the detected photon count rates provide sufficiently high statistics to allow real-time control of the longitudinal position of the Bragg-peak under clinical conditions.
\end{abstract}




\section{Introduction}

Heavy ion therapy is highly tumor-conformed and offers two main benefits over conventional radiation therapy: a more precise local dose distribution and an increased biological effectiveness in the target volume (Kraft 2000). Such a precision and effectiveness require accurate verification of the dose deposition and location. In fact, contrary to photons, ions with appropriate energy fully stop in the target. Thus, a mismatch between the planned and actual ion range could lead to severe over-dosage of the organs at risk or incomplete coverage of the tumor. For this reason, during treatment planning safety margins are applied around the tumor volume. To assess the adequacy of these margins, and possibly to reduce their size, a system that allows direct measurement of undesired variations in the ion range is still highly desirable.

So far, in-beam Positron Emission Tomography (PET) is the only method implemented clinically for in-situ verification of ion therapy. PET was used at the experimental treatment facility at GSI (Darmstadt, Germany) until 2008 (Parodi et al. 2008). During each irradiation, a PET activity map is acquired and compared, at the end of the fraction, with the one calculated on the basis of the treatment plan. If any discrepancies between the calculated and measured plans are detected, the radiotherapist can therefore estimate the mismatch between the delivered and planned dose and re-calculate the treatment plan for the next fraction (Enghardt et al. 2004). Furthermore one of the most important quantities deduced from PET monitoring is the primary particle range (Sommerer et al. 2006). Therapy verification would greatly benefit from an inbeam device capable to determine ion ranges in real-time. This is very unlikely to be done with PET due to the very low induced activity and to the radioisotopes half-lives, which are much longer than the characteristic time (of the order of a second) in which an iso-energy slice of tumor is treated, and that would correspond to the time available for the data acquisition.

To monitor the longitudinal position of the Bragg-peak in real-time, we already proposed a novel non-invasive technique that exploits the detection of prompt $\gamma$-rays issued from nuclear fragmentation (Testa et al. 2008). This technique is based on the fact that, within less than a nano-second following the ion beam impact on the target, photons and neutrons are emitted by excited nuclei formed each time a nuclear fragmentation process occurred. The highest majority of prompt photons arise from statistical decay of the slightly excited nuclei at energies below the nucleons binding energy ( 8MeV) (Riess 1989). Typically, Geant4 simulations suggest about 0.3 prompt photons to be emitted in average per incident C-ion at $300 \mathrm{MeV} / \mathrm{u}$ (Le Foulher et al. 2010). It is expected that the production of photons is correlated with the ion range, since fragmentation occurs almost all along the ion path except for the last 2-3 mm before the Braggpeak (Gunzert-Marx et al. 2008), where nuclear reaction cross sections decrease because the available energy in the projectile - target nucleus center of mass approaches the Coulomb 
barrier. This implies that, in principle, detection of the emitted prompt photons induced by primary and secondary ions could provide valuable information both on the dose distribution and on the Bragg-peak position. The latter point was first demonstrated by Min and co-workers, who could identify the Bragg-peak position with an accuracy of 1-2 mm for protons at 100 MeV (Min et al. 2006).

In an extension of their work we recently described a first experiment performed at GANIL (Caen, France) with $73 \mathrm{MeV} / \mathrm{u}{ }^{13} \mathrm{C}^{6+}$ ions (Testa et al. 2008, 2009), in which both target atoms and primary ions undergo nuclear fragmentation. It was shown that using a collimated detection set-up that provides information on the photon source-point location, and, consequently, on the Bragg-peak position, and a time-of-flight (TOF) set-up that discriminates prompt photons from the neutron background, would allow real-time monitoring with prompt $\gamma$-rays. Indeed, the use of TOF avoids bulky neutron shielding, since it allows reducing the corresponding background by at least one order of magnitude, depending on the beam time structure. Moreover, a possible gain of one order of magnitude in the detection solid angle as compared to the method proposed by Min and co-workers (Min et al. 2006) could be easily achievable covering a larger part of the azimuth around the patient by multiplying the number of collimated detectors.

The present paper reports on a new series of experiments that have been performed at the GANIL and GSI facilities with $95 \mathrm{MeV} / \mathrm{u}$ and $305 \mathrm{MeV} / \mathrm{u}{ }^{12} \mathrm{C}^{6+}$ ion beams. With these experiments we investigated whether a collimated set-up detecting single photons by means of TOF measurements can also be used at high energies that are typical for ion therapy. Moreover, the possibility to extract the same information from fast neutrons was also considered. Finally, the applicability of the technique both to cyclotron and synchrotron facilities is discussed. This represents a crucial issue since the proposed technique relies on TOF measurements that are strongly influenced by the beam time-structure. Based on the results obtained in the present study it is concluded real-time control of the longitudinal position of the Bragg-peak is possible under clinical conditions. 


\section{Materials and methods}

Figure 1 presents sketches of the two set-ups used in the GANIL and GSI experiments.

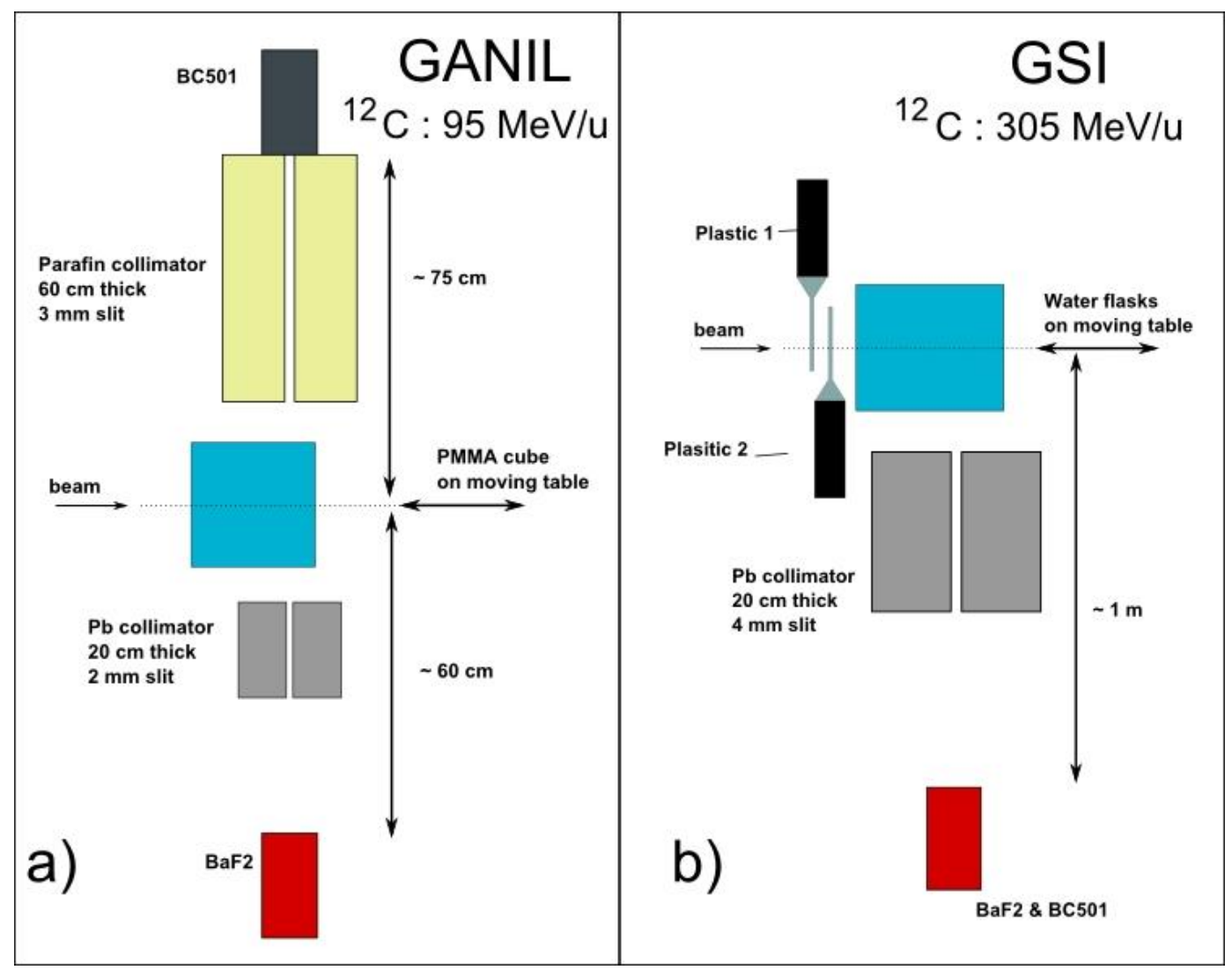

Fig 1 a) Diagram of the GANIL experimental set-up (95 MeV/u pulsed ${ }^{12} \mathrm{C}$ ion beam); b) Diagram of the GSI experimental set-up (305 MeV/u continuous ${ }^{12} \mathrm{C}$ ion beam)

In the experiment performed with low-energy carbon ions at the GANIL facility, carbon ions extracted from the vacuum beam line directly hit a cubic polymethyl methacrylate $\left(\mathrm{C}_{5} \mathrm{H}_{8} \mathrm{O}_{2}\right)_{n}$ (PMMA) target $\left(50 \times 50 \times 50 \mathrm{~mm}^{3}\right)$. At the GSI facility, higher energy ions bombarded a water target $\left(12 \times 25 \times 20 \mathrm{~cm}^{3}\right)$. In both experiments the targets were placed on a table that could be moved by remote-control along the beam axis (Fig. 1). Two detectors were used: a hexagonalshaped $\mathrm{BaF}_{2}$ scintillator (diameter: $9 \mathrm{~cm}$; thickness: $15 \mathrm{~cm}$ ) and a cylindrical liquid organic neutron scintillator (diameter: $5 \mathrm{~cm}$; thickness: $15 \mathrm{~cm}$; Saint Gobain BC501 model). The $\mathrm{BaF}_{2}$ scintillator was chosen for its excellent time response and its high efficiency for photon detection (due to its high-Z material composition), while the BC501 scintillator was used for its high efficiency for neutron detection and for the possibility to use pulse shape discrimination (PSD) (Barnabà et al. 1998). Indeed, since the shape of the signal produced in the liquid organic scintillator depends on the nature of the interacting radiation, the BC501 allows discrimination between signals arising from neutron or photon interactions. This was done according to the socalled charge comparison method (Normand et al. 2002) that consists in integrating, with a 
charge integrating analogue to digital converter (QDC) module, the anode signal of the BC501 detector over two integration gates shifted one with respect to the other. Figure 2 shows an example of pulse shape discrimination of signals acquired during the irradiation of the PMMA target at GANIL. Two aligned sets of points due to neutrons (up) and photons (down) interacting with the BC501 detector are clearly distinguishable.

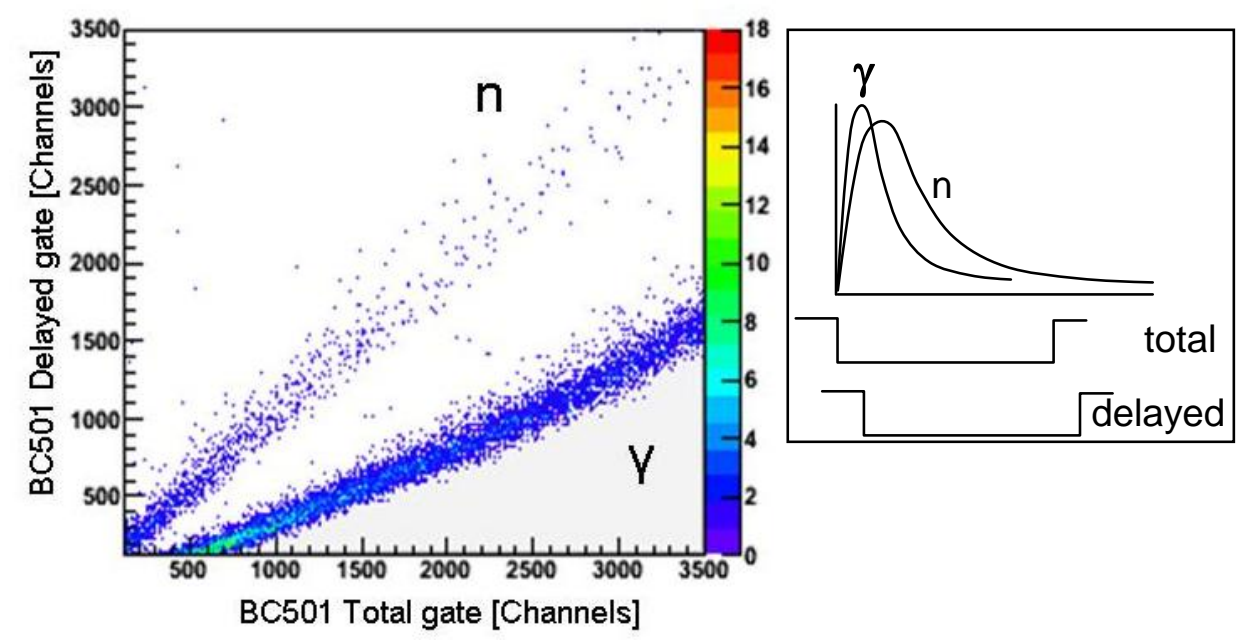

Fig 2 Pulse Shape Discrimination (PSD) applied to the BC501 anode signal. The signal is integrated over a delayed gate and a total integration gate as sketched in the right inset. The gate length is $300 \mathrm{~ns}$, the delay shift is $35 \mathrm{~ns}$. Two aligned sets of points corresponding to neutron (up) and photon (down) interactions are clearly distinguishable. The colour code of the histogram represents the density of the acquired data points

At GANIL, two different collimator materials (i.e., lead and paraffin) were used for the $\mathrm{BaF}_{2}$ and BC501 detectors respectively. This was done to optimize collimation of the photon component for the $\mathrm{BaF}_{2}$ detector, and to investigate a possible prompt neutron component with the BC501 detector. At GSI, a single lead collimator was used for both the $\mathrm{BaF}_{2}$ and $\mathrm{BC} 501$ detectors, which were stacked. Generally, a collimator can be characterized by the detection solid angle and the field of view which, in our case, corresponded to the ion-range segment from which photons can be detected. Both quantities, which are not straightforward to calculate for the set-up used here, have been evaluated by means of Geant 4 simulations, in order to take into account the shadowing effect around the edges of the collimator slits. To do so the experimental set-up was reproduced in a simulation in which a linear source of photons (with the same energy spectrum of prompt photons created during fragments de-excitation) replaced the PMMA or water target. As a result, the solid angle was $4.3 \times 10^{-4} \mathrm{sr}$ at GANIL and $4.5 \times 10^{-}$ ${ }^{4} \mathrm{sr}$ at GSI, while the field of view was 4.1 and $6.4 \mathrm{~mm}$ at GANIL and GSI, respectively.

The main difference between the two experiments is related to time pick-up measurements where the beam structure plays a major role. In fact, our monitoring technique rests upon the 
measurement of the time interval between the impact of the carbon ions on the target and the photon detection by the scintillators. At GANIL, where the beam is pulsed (beam pulse of $\sim 1 \mathrm{~ns}$ every $80 \mathrm{~ns}$ ), the cyclotron high-frequency (HF) signal (suitably delayed) could be used as stop signal. The start signal was provided either by the $\mathrm{BaF}_{2}$ or $\mathrm{BC} 501$ detection of a photon or neutron in an event-by-event acquisition mode. The choice of setting the lowest counting detectors as start signals was adopted to minimize the number of void events for which a start signal does not have a corresponding stop signal. The beam intensity was monitored by a $\mathrm{NaI}(\mathrm{Tl})$ detector $(10 \mathrm{~cm}$ in diameter and length; not shown in Fig. 1) placed at a large distance from the target, in order to obtain a counting rate proportional to the beam intensity but nearly independent of target position and collimation. This $\mathrm{NaI}(\mathrm{Tl})$ detector was calibrated with a Faraday cup at higher intensities. The beam intensity was set to about $1 \mathrm{nA}\left(10^{9} \mathrm{ions} / \mathrm{s}\right)$, in order to optimize the detector counting rates while avoiding pile-up and dead-time effects. In contrast, at the SIS-GSI synchrotron, where a continuum extraction mode was used ( $\sim \mathrm{s}$ extraction every $\sim 10 \mathrm{~s}$ ), the TOF stop signal was provided by two thin plastic scintillators intercepting the beam. During the carbon ion extraction, the intensity was kept at quite low values (a few $10^{5}$ ions/s), to allow an ion-by-ion triggering by the plastic scintillators (their efficiency was checked by comparing single and coincidence detection modes). These scintillators were also used to measure the integrated number of ions hitting the target.

\section{Results}

Figure 3 shows a two-dimensional spectrum of the energy deposited in the $\mathrm{BaF}_{2}$ detector as a function of the TOF, when the detector was looking at a region close to the Bragg-peak. 


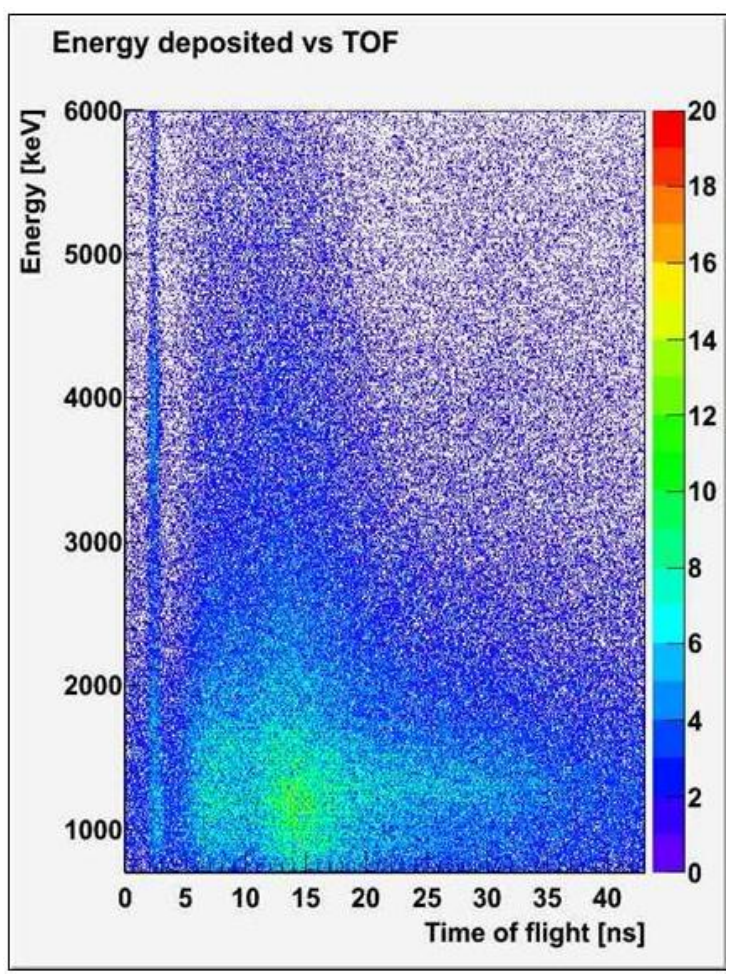

Fig 3 Two-dimensional spectrum of the energy deposited in the $\mathrm{BaF}_{2}$ detector as function of TOF. The spectrum was obtained at GANIL with the collimated detector looking at a target penetration depth of $16 \mathrm{~mm}$. The energy axis is calibrated for photons. The colour code of the histogram represents the density of the acquired data points.

Energy calibration was done using $\gamma$-emitting radioactive sources, and the time reference was set in a way that the origin of the time scale corresponds to the time when the carbon ions hit the target. Figure 3 shows a sharp prompt photon line at 2 ns having a continuous energy distribution that goes up to more than $6 \mathrm{MeV}$. The 2-D spectrum is dominated, however, by neutron-induced radiation which is detected after the prompt photon component and which contributes to a broad background noise. It is observed that selecting a photon energy above 2 $\mathrm{MeV}$ improves the signal-to-background ratio. It can be noticed as well that a time resolution of about $1 \mathrm{~ns}$ has been achieved with the $\mathrm{BaF}_{2}$ scintillator, which resulted in a much cleaner energy-TOF spectrum as compared to that reported in our previous study with a $\mathrm{NaI}(\mathrm{Tl})$ detector (Testa et al. 2009).

Figure 4 shows the TOF spectra obtained in the GANIL and GSI experiments. 


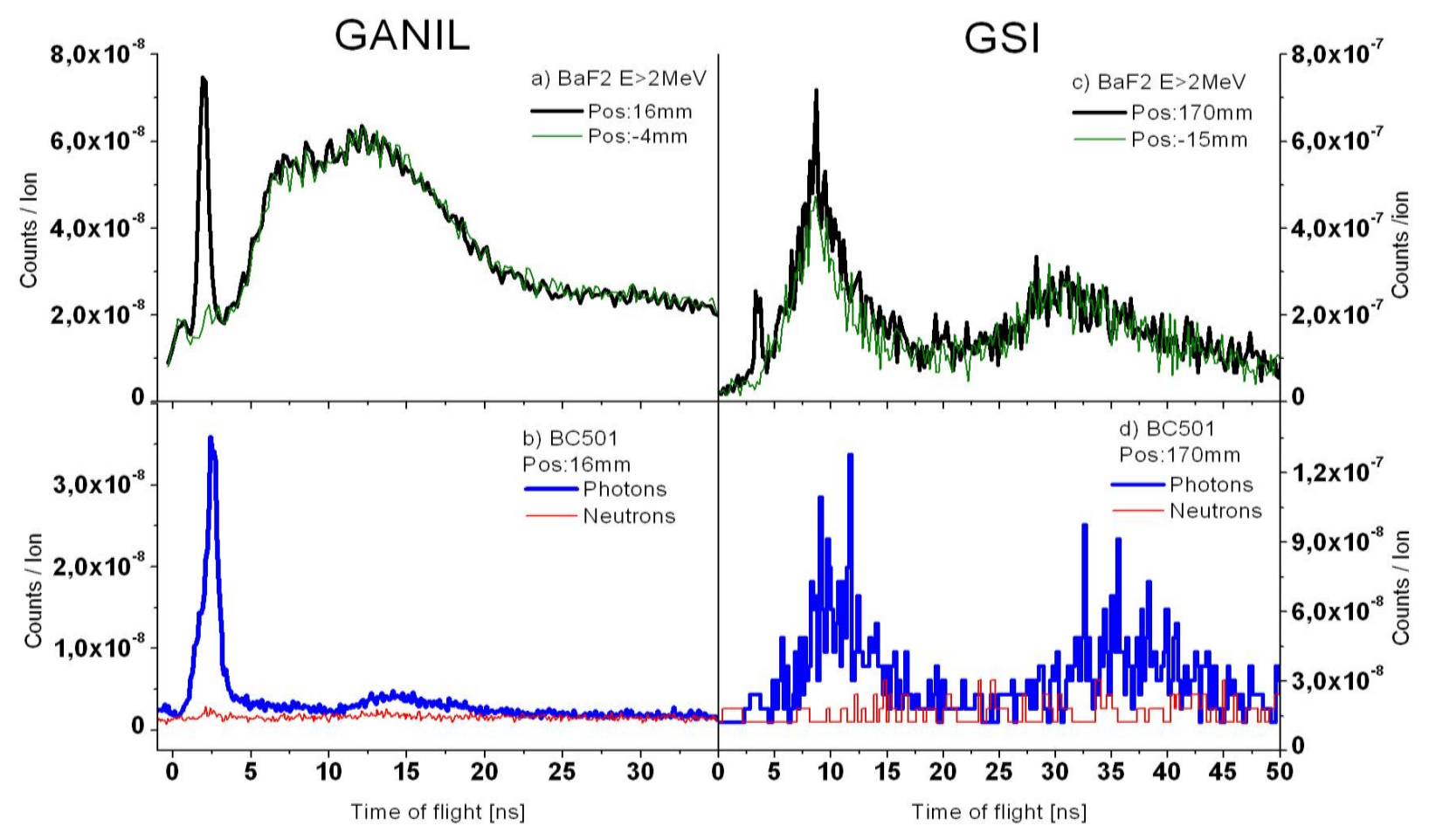

Fig 4 Left: TOF spectra for the GANIL (95 MeV/u pulsed ${ }^{12} \mathrm{C}$ ion beam) experiment. Right: TOF spectra for the GSI experiment (305 MeV/u continuous ${ }^{12} \mathrm{C}$ ion beam). The spectra are obtained for detector focussing on given target penetration depths ( $\mathrm{Pos}=0$ corresponds to the target entrance).

The upper part of the figure (Figs. 4a, 4c) shows the $\mathrm{BaF}_{2}$ TOF spectra for two different longitudinal detector positions: in front of the target entrance (thin lines) and close to the Braggpeak region (thick lines). In contrast, the lower part of the figure (Figs. 4b, 4d) shows the TOF spectra obtained with the BC501 detector for a position close to the Bragg-peak, depending on the nature of the detected particle: photon (thick lines) and neutron (thin lines). As in Fig. 3, the time reference was set in a way that the origin of the time scale corresponds to the time when the carbon ions hit the target. Figs. $4 \mathrm{a}$ and $4 \mathrm{c}$ clearly show that the prompt photon peak arising at $2 \mathrm{~ns}$ (GANIL) and at 3-4 ns (GSI) completely disappears when the collimated detector is not focused on the ion path region. It is therefore concluded that these prompt-peak photons have reached the detector after passing through the collimator slit without undergoing any interaction.

In Fig. 4a, a broad distribution is present between 5 and 20 ns. Actually this broad distribution consists of two components hardly distinguishable in the figure: one between 5 and $10 \mathrm{~ns}$, and the other between 10 and $20 \mathrm{~ns}$. The relative integrals of these two components depend strongly on the energy threshold applied ( $2 \mathrm{MeV}$ in Fig. 4a). We attribute the first component to photons created through $(\mathrm{n}-\gamma)$ reactions in the lead collimator. Two pieces of evidence confirm this hypothesis: i) no structure is present between 5 and $10 \mathrm{~ns}$ in the BC501 (which did not include a 
lead collimator) TOF spectrum (Fig. 4b), ii) at GSI, where both detectors included a lead collimator, the TOF spectra look very similar for both $\mathrm{BaF}_{2}$ and BC501 (Figs. 4c and 4d). The second component in Fig. 4a at 10-20 ns probably comes from photons that have scattered or were produced by neutrons through $(\mathrm{n}, \gamma)$ reactions in the walls of the experimental cave. The same structure is present in the photon TOF spectrum of Fig. $4 \mathrm{~b}$ but its intensity is attenuated, due to the low radial detection efficiency of the BC501 detector (5cm in diameter). Moreover, as already mentioned, the relative intensity of this component strongly depends on the energy threshold applied to the spectra. The lower the energy threshold, the higher is the number of photons scattered at the walls of the cave. With no energy cut, the component at 10-20 ns is higher than that at 5-10 ns (not shown).

With PSD, neutrons and photons detected by the BC501 scintillator (Barnabà et al. 1998, Normand S. et al. 2002) could be distinguished (Figs. 4b and 4d). At $90^{\circ}$ with respect to the beam direction, where the detectors were placed, photons and neutrons overwhelm all the charged particles produced during ion fragmentation. In Fig. $4 \mathrm{~b}$ one can notice that the prompt photon peak arising at about $2.5 \mathrm{~ns}$ is broader than that in the $\mathrm{BaF}_{2}$ TOF spectrum. This is mainly due to the poorer time resolution of the BC501 scintillator and to the photon collimation, which was not optimal because paraffin was specifically used to investigate any prompt neutrons. Additionally, the neutron component of the TOF spectrum is completely flat (Fig. 4b) similar to that obtained for the GSI measurements (Fig. 4d). Interestingly, no prompt photon peak is visible in the GSI BC501 spectrum (Fig. 4d). This is due to the low statistics accumulated for each target position and to a much poorer photon detection efficiency of the BC501 detector compared to that of the $\mathrm{BaF}_{2}$ detector.

The $\mathrm{BaF}_{2}$ TOF spectrum shown in Fig. 4c (GSI) is rather similar to the one presented in Fig. 4a (GANIL) although the statistics was one hundred times lower at GSI, due to the limited beam time and the low beam intensity required for single ion triggering with the plastic scintillators. The relative intensity between the prompt photon peak and the photon distribution induced by neutron interactions in the lead collimator (arising from 5 to $15 \mathrm{~ns}$ ) appears inverted compared to that in Fig. 4a. This is mainly because the total neutron production rate during fragmentation is much higher at $305 \mathrm{MeV} / \mathrm{u}$ than at $95 \mathrm{MeV} / \mathrm{u}$, due to the larger range of $305 \mathrm{MeV} / \mathrm{u}$ carbon ions in matter. The time difference between the prompt peak and the third bump (wall-scattered background photons, 25 to $40 \mathrm{~ns}$ ) can be explained by the geometrical dimensions of the experimental cave which is larger at GSI than at GANIL. 


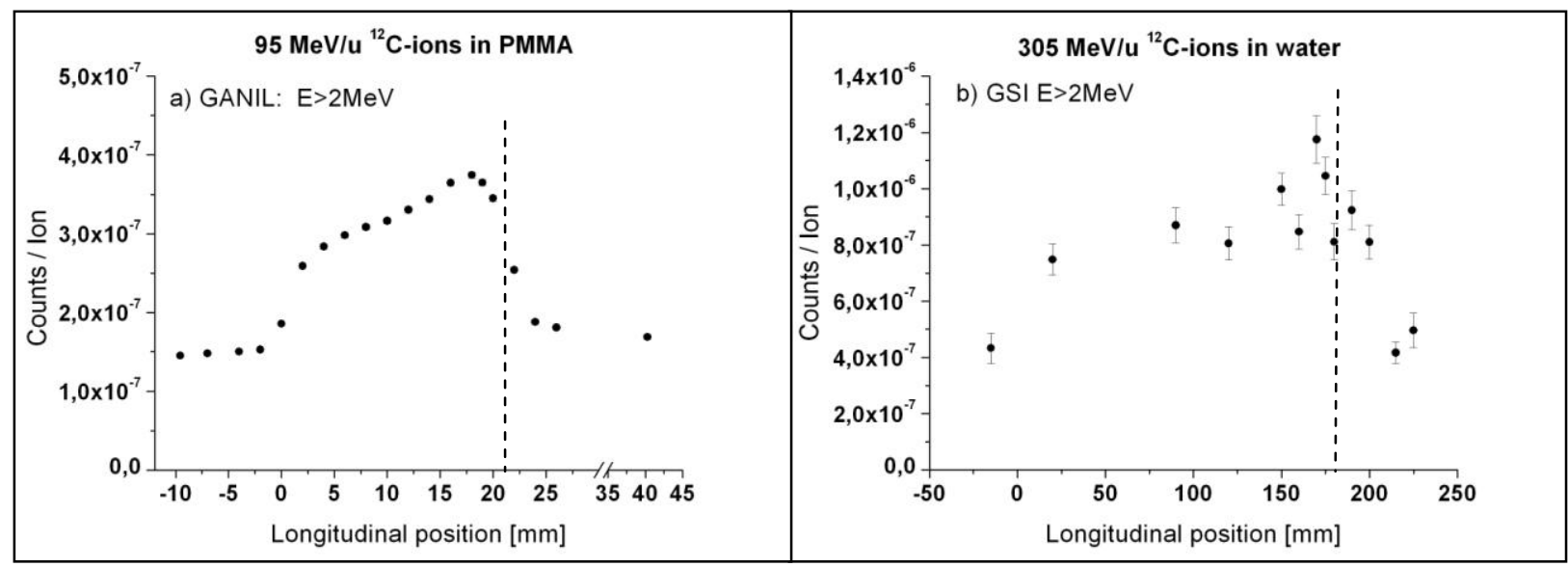

Fig 5 a) Scan GANIL; (b) Scan GSI. The origin of the longitudinal axis corresponds to the target entrance position. The calculated Bragg-peak position is given by the dashed vertical line. The error bars correspond to the statistical errors only.

The scan profiles presented in Fig. 5 were obtained by integrating the counts detected by the $\mathrm{BaF}_{2}$ scintillator in the prompt photon peak of the TOF spectra (see Figs. 4a and 4c) at various longitudinal positions from upstream the target entrance to downstream the Bragg-peak. For both experiments, the time integration interval was $1.5 \mathrm{~ns}$ centered on the prompt photon peak. As mentioned above, a photon energy threshold of $2 \mathrm{MeV}$ was chosen to optimize the statistics and the signal-to-background ratio. A clear correlation is observed in both cases between the ion path and the photon production yield. A decrease by a factor of about 2 is seen close to the Bragg-peak position, as reported previously for low-energy ions (Testa et al. 2008, 2009). The increase of the $\gamma$-ray yield at the end of the ion path can be attributed to an increase of the fragmentation cross section, when the ion energy decreases.

\section{Discussion and Conclusion}

No evidence for a prompt neutron component correlated with the primary ion range was found for the GANIL experiment, where a dedicated paraffin collimation was used. Therefore, fast neutrons detected at $90^{\circ}$ cannot be considered to provide useful information on the dose profile. However, this does not imply that neutrons could not provide some information at a more forwarded angle, but this remains to be investigated in another study. The PSD technique used with the BC501 detectors nevertheless allowed a better understanding of the shape and components of the $\mathrm{BaF}_{2}$ TOF spectra, in both experiments.

The major requirements on the measured $\gamma$-ray profiles are a spatial resolution of about 1-2 mm and a clear difference between the region before and after the Bragg-peak. Based on the results of the present paper, prompt photon detection appears a very promising technique to be used for 
a real-time ion-range monitor. Indeed, for a prompt photon energy threshold of $2 \mathrm{MeV}$, we obtained along the ion path, in both experiments, a net count rate per incident carbon ion, unit solid angle and unit path length of $1 \times 10^{-7}$ photons/(ion $\times$ msr $\times m m$ ). The normalisation over the solid angle and the field of view was done according to the values reported in the materials and methods section that were obtained by means of Geant4 simulations. For comparison, the background count rate slightly depended on the experimental set-up and was about $1-2 \times 10^{-7}$ photons/(ion $\times$ msr $\times$ mm) If we consider as an example a patient treatment plan in which $7 \times 10^{8}$ carbon ions are required to deliver an absorbed dose of $1 \mathrm{GyE}$ to a tumour volume of $120 \mathrm{~cm}^{3}$, divided in 39 slices each $3 \mathrm{~mm}$ wide (Kraemer et al. 2000), there are on average $1.8 \times 10^{7}$ carbon ions per slice. According to the above-mentioned values we would obtain, with the single $\mathrm{BaF}_{2}$ detector as used in the present experimental configuration and a photon threshold of $2 \mathrm{MeV}$, about $7 \gamma$-counts within and $3 \gamma$-counts outside the ion-path for the GANIL scan shown in Fig. $5 \mathrm{a}$, while we would obtain about $20 \gamma$-counts within and $7 \gamma$-counts outside the ion path for the GSI scan shown in Fig. 5b. Note that the signal-to-background ratio measured in the present case is not yet optimized: more than $90 \%$ of the 1 litre volume of $\mathrm{BaF}_{2}$ detects only background, which requires a large quantity of lead shielding to be used, which in turn creates a high neutron and gamma background. It is therefore concluded that, with a possible increase of the solid angle and detection efficiency by one or two orders of magnitude, the statistics would be sufficient to determine the Bragg-peak location for each slice with an accuracy of about $1 \mathrm{~mm}$. With an intensity of $10^{8}$ ions/s, this information can be provided within the time of around 200 $\mathrm{ms}$ required to irradiate the slice volume. Thus, the clinician would be able to interrupt the treatment fraction immediately in case of mispositioning. Moreover, we have demonstrated here that the different beam time-structures (pulsed or continuous) do not preclude TOF measurements with sub-nanosecond precision. In case of a continuous spill structure the main requirement for TOF systems is to allow identification of the primary ions one by one, while in case of a pulsed spill structure the time resolution of TOF systems has to be of the same order as the pulse duration. Thus, our technique may be applicable both at cyclotrons if the pulse time-length is about $1 \mathrm{~ns}$, and at synchrotrons if a detector is available that allows to trigger a high counting rate. Such a detector is currently being developed at our laboratory.

Note that the present system is based on moving a target in front of a fixed collimator. In clinical operation conditions, the gamma camera should be moveable, which is made possible by using relatively compact shielding, thanks to the TOF technique. Moreover, the use of several collimated detectors focussing on different positions in the patient would allow detection of the dose profile along the primary ion range without moving the patient.

It is concluded that, based on the results of the two experiments described here, single photon emission tomography including a collimated set-up and TOF measurements constitutes a 
promising method to control the dose distribution at high energies during ion therapy in situ and in real-time.

Acknowledgements: Part of this work was funded by the Rhône-Alpes regional research program for ion therapy ETOILE. 


\section{References}

Barnabà O., Chen Y.B., Musitelli G., Nardò R., Raselli G.L., Rossella M., Torre P. (1998) A full-integrated pulse shape discriminator for liquid scintillator counters Nucl. Instr. Meth. A $410220-228$

Enghardt W., Parodi K., Crespo P., Fiedler F., Pawelke J., Pönisch F. (2004) Dose quantification from in-beam positron emission tomography Radioth. Oncol. 73 96-8

Gunzert-Marx K., Iwase H., Schardt D. Simon R. S. (2008) Secondary beam fragments produced by $200 \mathrm{MeV} / \mathrm{u} 12 \mathrm{C}$ ions in water and their dose contributions in carbon ion radiotherapy New Journal of Physics 10

Kraemer M. Jakel O., Haberer T., Kraft G., Schardt D., Weber U. (2000) Treatment planning for heavy-ion radiotherapy: physical beam model and dose optimization Phys. Med. Biol. 45 $3299-3317$

Kraft G. (2000) Tumor therapy with heavy charged particles Prog. Part. Nucl. Phys. 45 S473544

Le Foulher F., Bajard M., Chevallier M., Dauvergne D., Freud N., Henriquet P., Karkar S., Létang J.M, Plescak R., Ray C., Schardt D., Testa E., Testa M. (2010) Monte Carlo simulations of prompt-gamma emission during carbon ion irradiation. Accepted for publication in IEEE Transactions on Nuclear Science

Min C.-H., Kim C. H. Youn M.-Y., Kim J.-W. (2006) Prompt gamma measurements for locating the dose falloff region in proton therapy Appl. Phys. Lett. 89

Normand S., Mouandab B., Haana S., Louvel M. (2002) Discrimination methods between neutron and gamma rays for boron loaded plastic scintillators Nucl. Instr. Meth. A 484 342-350 Parodi K., Bortfeld T, Enghardt W., Fiedler F., Knopf A., Paganetti H., Pawelke J., Shakirin G., Shih H. (2008) PET imaging for treatment verification of ion therapy: Implementation and experience at GSI Darmstadt and MGH Boston Nucl. Instr. Meth. A 591 282-286

Riess S. (1989) Exclusive photon yields from peripheral collisions of ${ }^{40} \mathrm{Ar}+{ }^{158} \mathrm{Gd}$ at $44 \mathrm{MeV} / \mathrm{u}$ Nucl. Phys. A495 49c-56c

Sommerer F., Parodi K., Ferrari A., Poljanc K., Enghardt W., Aiginger H. (2006) Investigating the accuracy of the FLUKA code for transport of therapeutic ion beams in matter Phys. Med.

Biol. $514385-4398$

Testa E., Bajard M., Chevallier M., Dauvergne D., Le Foulher F., Freud N., Létang J.-M., Poizat J.-C., Ray C., Testa M. (2008) Monitoring the Bragg peak location of $73 \mathrm{MeV} / \mathrm{u}$ carbon ions by means of prompt gamma ray measurements Appl. Phys. Lett. 93

Testa E., Bajard M., Chevallier M., Dauvergne D., Le Foulher F., Freud N., Létang J.-M., Poizat J.-C., Ray C., Testa M. (2009) Dose profile monitoring with carbon ions by means of prompt-gamma measurements Nucl. Instr. Meth. Phys. Res., B 267 pp. 993-996 